\title{
Zero-field and Larmor spinor precessions in a neutron polarimeter experiment
}

\author{
S. Sponar ${ }^{a}$, J. Klepp ${ }^{a}$, G. Badurek ${ }^{a}$, and Y. Hasegawa ${ }^{a, b *}$ \\ ${ }^{a}$ Atominstitut der Österreichischen Universitäten, Stadionallee 2, A-1020 Vienna, \\ Austria \\ ${ }^{b}$ PRESTO, Japan Science and Technology Agency (JST), Kawaguchi, Saitama \\ 332-0012, Japan
}

\begin{abstract}
We present a neutron polarimetric experiment where two kinds of spinor precessions are observed: one is induced by different total energy of neutrons (zero-field precession) and the other is induced by a stationary guide field (Larmor precession). A characteristic of the former is the dependence of the energy-difference, which is in practice tuned by the frequency of the interacting oscillating magnetic field $\omega_{R}$. In contrast the latter completely depends on the strength of the guide field, namely Larmor frequency $\omega_{\mathrm{L}}$. Our neutron-polarimetric experiment exhibits individual tuning as well as specific properties of each spinor precession, which assures the use of both spin precessions for multi-entangled spinor manipulation.
\end{abstract}

Key words: neutron, spin precession, Larmor precession, zero-field precession, polarimeter

* Corresponding author Phone +43-1-58801-14190; Fax +43-1-58801-14199.

Email address: Hasegawa@ati.ac.at (Y. Hasegawa $\left.{ }^{a, b}\right)$. 
In the last years great effort has been devoted to the development of quantum information and communication technology [1]. Among them creation and manipulation of entanglement is a key issue and experimental realizations with the use of photons, atoms, ions, nuclear-magnet, superconducting qubits etc. have been reported [2]. There, the entanglement between spatially separated systems are mainly used to exploit non-local property of quantum systems. In contrast, investigations of two-qubit entanglement with neutrons, one of the most useful quantum system to be utilized for studies of quantum mechanical phenomena [3], have been performed with the use of their degrees of freedom, namely spin and path, in a single particle: a violation of a Bell-like inequality [4], contextual nature of quantum theory [5], and a full tomographic analysis of Bell-states [6] were carried out.

For further development towards multi-entanglement, it is inevitable to establish manipulation of other degrees of freedom. It is know that the total energy of neutrons can be manipulated with the use of interactions between a neutron's magnetic moment and a time-dependent oscillating magnetic field [7,8,9]. Recently, we have accomplished an experiment to exhibit a coherent energy manipulation of neutrons with the use of radio-frequency (RF) oscillating magnetic field [10], enabling neutrons to be applied for investigations of multi-entanglement. Thus, we find it significant to demonstrate explicitly a phase manipulation for different energy eigenstates which will be utilized in forthcoming experiments such as a demonstration of triple-entanglment with a GHZ-state [1]. In the neutron scattering community, a spin precession, alternatively a phase shift, due to energy difference is known as zero-field precession [12,13] and utilized for zero-field spin-echo spectroscopy [14,15,16,17].

When a neutron is exposed to a stationary magnetic field, the motion of its 
spin vector is thoroughly described by the Bloch-equation exhibiting Larmor precession:

$$
\frac{d \vec{S}}{d t}=\gamma \vec{S} \times \vec{B}
$$

where $\gamma$ is the gyromagnetic ratio given by $\mu /|S|, \mu$ and $\vec{S}$ are magnetic moment and the neutron's spin, respectively. This is the equation of motion of a classical magnetic dipole in a magnetic field, which shows the precession of the spin vector $\vec{S}$ about the magnetic field $\vec{B}$ with the Larmor frequency $\omega_{\mathrm{L}}=|2 \mu B / \hbar|$. It is worth noting here that the Larmor precession angle (rotation angle) is obtained with a frequency $\omega_{\mathrm{L}}$ and the propagation time $\mathrm{T}$ as $\omega_{\mathrm{L}} \mathrm{T}$, solely depends on the strength of the applied magnetic field. In practical experiments, the Larmor precession is utilized by a variety of DC spin rotators.

In contrast, when a neutron interacts with a time-dependent, rather oscillating, magnetic field, photon exchange occurs and the total energy of neutrons is shifted. In particular when a so-called resonant spin-flipper is activated, the neutron emits (or absorbs) a photon of energy $\hbar \omega_{R}$, thus the total energy of the neutron decreases (or increases) by $\hbar \omega_{\mathrm{R}}$ during the interaction [9]. As a consequence, an additional phase, a zero-field phase, $\omega_{\mathrm{R}} \mathrm{T}$ is accumulated during the propagation afterwards. This phase shift, alternatively a spin precession, due to energy shift emerges even without a guide field, thus it is referred to a zero-field precession in literature [12,13]. It should be emphasized that this zero-field phase is independent of the applied guide field, namely the Larmor phase $\omega_{\mathrm{L}} \mathrm{T}$, and purely depends on the frequency of the spin-flipper. In the following neutron polarimeter experiments, we demonstrate how one can tune 
a zero-field phase $\omega_{R} \mathrm{~T}$ and a Larmor phase $\omega_{\mathrm{L}} \mathrm{T}$ independently.

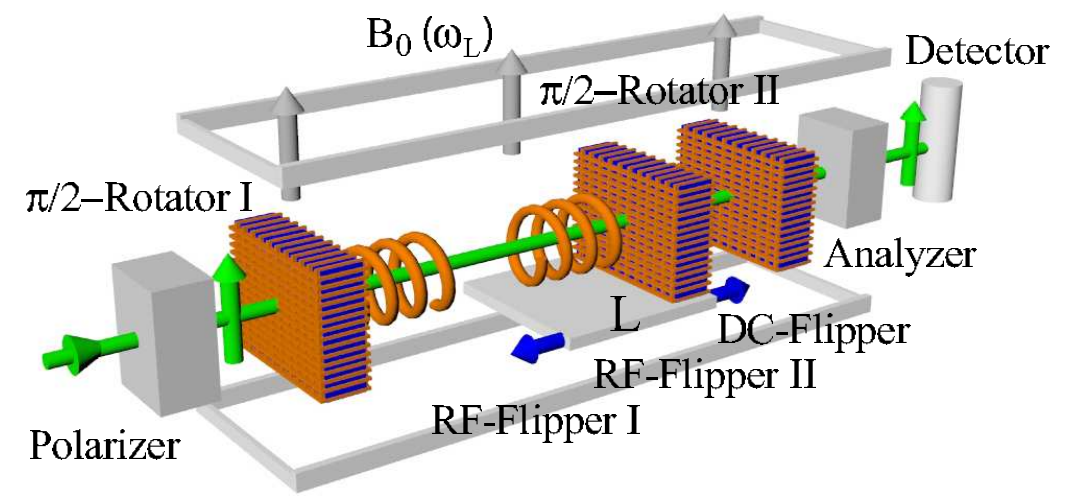

Fig. 1. Experimental setup for demonstrations of zero-field and Larmor spin precessions. From a polarized incident beam the first $\pi / 2$ spin-rotator generates a superposition of $| \pm z\rangle$. The first RF flipper induces the energy shift $\hbar \omega_{R}$ for each flipped spin eigenstate $|\mp z\rangle$, which leads to the zero-field spin precession until the second RF flipper where the energy difference is compensated. The guide field $B_{0}$, permeated along the beam path, leads to the Larmor spin precession. The second $\pi / 2$ spin-rotator together with the analyzer enables to exhibit the intensity modulation due to the spin precessions. The translation of the DC and the RF flippers manipulate individually the two phases when the RF flipper is turned on and off.

The experiments were carried out at the neutron polarimetry facility at the 250-kW TRIGA research reactor of the Atomic Institute of the Austrian Universities in Vienna [18,19,20,21]. A schematic view of the experimental setup is shown in Fig.1. The incident neutron beam is monochromatized (with a mean wave length of $\lambda=1.99 \AA$ which corresponds to a velocity of $1990 \mathrm{~m} / \mathrm{s}$ ) by the use of a pyrolitic graphite crystal and polarized (average degree of polarization over $98 \%$ ) by reflection from a bent Co-Ti supermirror array. The diameter of the beam is confined to about $4 \mathrm{~mm}$ in diameter by a $\mathrm{Cd}$ diaphragm. The initial polarization vector $\vec{P}_{\mathrm{i}}$ is perpendicular to the beam trajectory and defines the $+\hat{\mathbf{z}}$-direction. Another suppermirror array is used to analyze the final 
polarization $\vec{P}_{\mathrm{f}}$. Depolarization of the neutron beam is minimized by applying a guide field along the beam trajectory wherever necessary: this guide field $B_{0}$ determines the Larmor frequency $\omega_{\mathrm{L}}$.

In a neutron polarimeter setup described above, after going through a $\pi / 2$ spin-rotator, an incident polarization is interpreted as superposition of two orthogonal spin states $| \pm z\rangle$ and different phase shifts of these two states under further spinor manipulations result in a polarization change of the emerging neutron beam, i.e., we take a neutron polarization-interference scheme. Between the two $\pi / 2$ spin-rotators, three spin-flippers are placed in the beam: the first two are RF spin-flippers followed by a DC spin-flipper. In addition, one of the RF and the DC flippers are mounted together on a single translator, allowing to tune the propagation time of neutrons between the RF spin-flippers. The polarization vector after passing through all spin-flippers is expected to lie in the xy-plane, resulting from the interference between the states $| \pm z\rangle$. A spin analyzer together with the second $\pi / 2$ spin-rotator is used to resolve the different phase shifts accumulated through spin-flips.

The first experiment shows a pure Larmor precession: both RF flippers are turned off and only the DC flipper is in operation. In this case, the superposed states $| \pm z\rangle$ (and the flipped states $|\mp z\rangle$ later) simply propagate within the guide field $B_{0}$. These states $|+z\rangle$ and $|-z\rangle$ are the eigenstates of the guide field: no additional spinor rotation occurs. Then, each state merely obtains a Larmor phase $\alpha$ due to the guide field, which is given by

$$
\begin{aligned}
\alpha & =\omega_{\mathrm{L}}\left(\mathrm{T}_{1}-\mathrm{T}_{2}\right) \\
& =\omega_{\mathrm{L}}\left(\left(\mathrm{T}_{1}^{0}+\Delta \mathrm{T}\right)-\left(\mathrm{T}_{2}^{0}-\Delta \mathrm{T}\right)\right) \\
& =\alpha_{0}+2 \omega_{\mathrm{L}} \Delta \mathrm{T},
\end{aligned}
$$



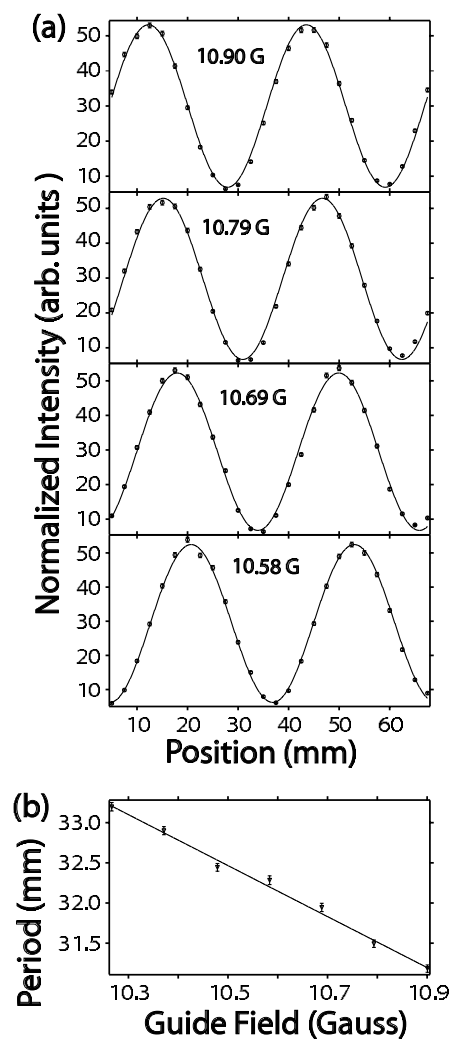

Fig. 2. (a) Typical intensity oscillations with least square fits for the Larmor spin precession. Curves are shown with the guide field $B_{0}=10.90,10.79,10.69$, 10.58G.(b) Dependence of the period on the strength of the guide field depicted for seven values of the guide field. A clear linear dependence agrees well with the theoretical predictions (see, Eq. (2)).

with $\alpha_{0}=\omega_{\mathrm{L}}\left(\mathrm{T}_{1}^{0}-\mathrm{T}_{2}^{0}+\mathrm{T}_{3}^{0}\right) . \mathrm{T}_{1}, \mathrm{~T}_{2}, \mathrm{~T}_{\mathrm{j}}^{0}$ and $\Delta \mathrm{T}$ are the propagation time before and after the DC flipper, the propagation time at the initial position, and the time shift for $\mathrm{T}_{1}, \mathrm{~T}_{2}$ by shifting the position of the $\mathrm{DC}$ flipper. After the beam passes the $\pi / 2$ spin-rotator and the analyzer, clear sinusoidal intensity oscillations of high contrast were obtained. Typical experimental data with least square fits are shown in Fig.2(a): the strength of the guide field was varied at $B_{0}=10.90,10.79,10.69,10.58 \mathrm{G}$. The shift of the oscillations is due to $\omega_{\mathrm{L}} \mathrm{T}$ (see, $\alpha_{0}$ in Eq.(2)). In addition, the dependence of the period of the oscillations on the strength of the guide field is plotted in Fig.2(b). A linear 
dependence, of which inclination is in good agreement with the theoretically predicted value, is seen, which confirms the precession angle given by Eq.(2).
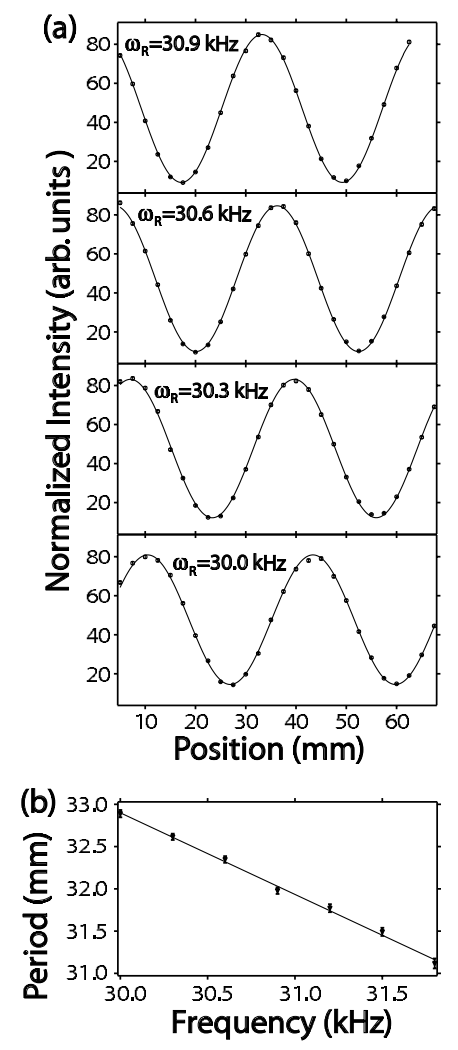

Fig. 3. (a) Typical intensity oscillations with least square fits for the zero-field spin precession. Curves are shown at the RF flipper frequencies $\omega_{R}=30.9,30.6$, 30.3, $30.0 \mathrm{kHz}$. (b) Dependence of the period on the frequency depicted for seven values of the frequency. A clear linear dependence agrees well with the theoretical predictions (see, Eq. (3)).

Next, characteristics of the zero-field precession are considered: both RF flippers are turned on. In this case, the superposed states $| \pm z\rangle$ is once flipped to $|\mp z\rangle$, and finally again flipped back to $| \pm z\rangle$. Then, the spin precession angle $\alpha^{\prime}$ is expected to be given by the propagation time, $T_{1}, T_{2}$, and $T_{3}$ after each spin flipper by 


$$
\begin{aligned}
\alpha^{\prime} & =\omega_{\mathrm{L}}\left(\mathrm{T}_{1}-\mathrm{T}_{2}+\mathrm{T}_{3}\right)+\omega_{\mathrm{R}} \mathrm{T}_{1} \\
& =\omega_{\mathrm{L}}\left\{\left(\mathrm{T}_{1}^{0}+\Delta \mathrm{T}\right)-\mathrm{T}_{2}^{0}+\left(\mathrm{T}_{3}^{0}-\Delta \mathrm{T}\right)\right\}+\omega_{\mathrm{R}}\left(\mathrm{T}_{1}^{0}+\Delta \mathrm{T}\right) \\
& =\alpha_{0}^{\prime}+\omega_{\mathrm{R}} \Delta \mathrm{T},
\end{aligned}
$$

with $\alpha_{0}^{\prime}=\omega_{\mathrm{L}}\left(\mathrm{T}_{1}^{0}-\mathrm{T}_{2}^{0}+\mathrm{T}_{3}^{0}\right)+\omega_{\mathrm{R}} \mathrm{T}_{1}^{0}$. In this configuration, no Larmor precession is expected to be induced by the change of $\Delta \mathrm{T}$, since a positive and a negative change of $\mathrm{T}_{1}$ and $\mathrm{T}_{3}$ completely compensate each other by shifting the position of the DC flipper and the second RF flipper. In order to prove the frequency dependence of the precession, the frequency of the RF flippers were varied with keeping the strength of the guide field by $B_{0}=10.59 \mathrm{G}$. Typical intensity modulations are shown in Fig.3(a) at the RF flipper frequencies of $\omega_{\mathrm{R}}=30.9$, 30.6, 30.3, and $30.0 \mathrm{kHz}$. Clear sinusoidal oscillations of high contrast were again obtained. A slight reduction of the amplitude of the obtained oscillations is solely due to the detuning of the frequency $\omega_{R}$ in the vicinity of the resonance for the flip-mode. And the shift of the oscillations arises from $\omega_{\mathrm{R}} \mathrm{T}$ (see, $\alpha_{0}^{\prime}$ in eq.(3)). In addition, a dependence of the period on the frequency is plotted in Fig.3(b). A linear dependence is seen and its inclination is in good agreement with the theory, which confirms the precession angle given by Eq.(3).

Note that the setup is constructed in a way that no spin rotation due to the Larmor precession will occur, since the Larmor precessions before and after the second RF flipper, i.e., $\Delta \mathrm{T}_{1}$ and $\Delta \mathrm{T}_{3}$, are compensated each other with all flippers turned on (see Eq.(3)). This independence of the Larmor precession can also be accessed in our experimental setup. In particular, the frequency of the RF flippers were tuned at the resonance $\omega_{0}=31.8 \mathrm{kHz}$ and the strength of the guide field was varied at $B_{0}=10.90,10.79,10.69,10.58 \mathrm{G}$. Typical experimental data with least square fits are shown Fig.4(a): identical sinusoidal oscillations are obtained, confirming no spin rotation due to Larmor 

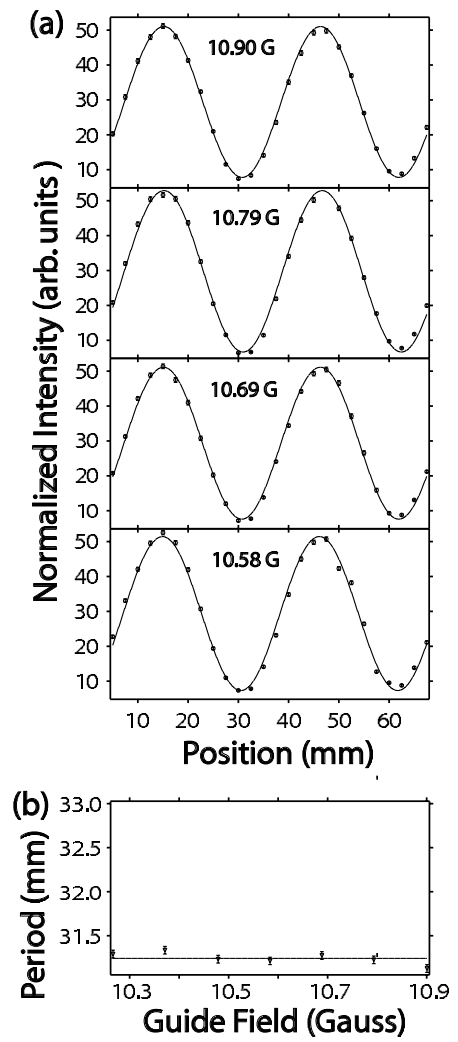

Fig. 4. (a) Typical intensity oscillations with least square fits for the zero-field precession. Curves are shown with the guide field $B_{0}=10.90,10.79,10.69,10.58 \mathrm{G}$. All curves are identical as expected. (b) The period is plotted versus the strength of the guide field for seven values. It is constant and independent of the strength of the guide field.

precessions. In addition, the period is plotted versus the strength of the guide field in Fig.4(b). Independent behavior of the period from the field strength is seen as expected by Eq.(3).

The results are in good agreement with theoretically predicted behavior: the frequency of the oscillating fields affects only on the zero-field precession while the strength of the field does on the Larmor precession. The tactics, that the $\mathrm{RF}$ and the DC flippers are displaced by the same amount, allows individual control of both precessions. This is a great advantage for the separate (phase) manipulation of the two-spaces, i.e., spin and energy spaces, in future neutron 
optical experiments. We are now proceeding further neutron interferometric and polarimetric experiments, where multi-entanglement in a single neutron system will be investigated, in particular, with the use of entanglement between degrees of freedoms such as path, spin, and energy.

In summary, we have presented experiments with a neutron polarimeter where two spin-precessions are manipulated to show their characteristics. The zerofield precession is independent of the strength of the guide field and purely depends on the frequency of the RF spin-flippers, where an energy shift occurs. In contrast, the Larmor precession solely depends on the strength of the applied magnetic field. In addition, we have exhibited a method to manipulate individually the two phases, resulting from each spin precession. The method used here will be utilized in further neutron optical experiments dealing with multi-entanglement in a single particle system.

This work has been partly supported by the Austrian Science Foundation, FWF (P17803-N02). Y.H. would like to thank the Japan Science and Technology Agency (JST) for financial support.

\section{References}

[1] M.A. Nielsen and I. Chuang, Quantum Computation and Quantum Information, (Cambridge Unviversity Press, Cambridge, 2000).

[2] S. Haroche and J. Raimond, Exploring the Quantum, (Oxford Unviversity Press, Oxford, 2006).

[3] H. Rauch and S.A. Werner, Neutron Interferometry, (Clarendon Press, Oxford, 2000). 
[4] Y. Hasegawa, R. Loidl, G. Badurek, M. Baron, and H. Rauch, Nature 425, 45 (2003).

[5] Y. Hasegawa, R. Loidl, G. Badurek, M. Baron, and H. Rauch, Phys. Rev. Lett. 97, $230401(2006)$.

[6] Y. Hasegawa, R. Loidl, G. Badurek, S. Filipp, J. Klepp, and H. Rauch, Phys. Rev. A 76, 052108 (2007).

[7] B.Alefeld, G. Badurek, and H. Rauch, Z. Phys B41 231 (1981).

[8] G. Badurek, H. Rauch, and J. Summhammer, Phys. Rev. Lett. 51, 1015 (1983).

[9] J. Summhammer, Phys. Rev. A 47, 56 (1993).

[10] S. Sponar, J. Klepp, R. Loidl, S. Filipp, G. Badurek, Y. Hasegawa, and H. Rauch, submitted to Phys. Rev. Lett.

[11] D.M. Greenberger, M.A. Horne, and A. Zeilinger, in Bell's Theorem, Quantum Theory, and Concepts of the Universe, edited by M.Kafatos (Kluwer Academics, Dordrecht, The Netherlands, 1989).

[12] R. Golub, R. Gähler, and T. Keller, Am. J. Phys. 62779 (1994).

[13] W.H. Kraan, S.V. Grigoriev, R. kreuger, F.M. Mulder and M.Th. Rekveldt, Physica B297, 23 (2001).

[14] R. Gähler, and R. Golub, Z. Phys. B65 269 (1987).

[15] R. Gähler, and R. Golub, Phys. Lett. A 12343 (1987).

[16] D. Yamazaki, Nucl. instr. meth. A 488, 623 (2002).

[17] S.V. Grigoriev, W.H. Kraan, and M.Th. Rekveldt, Phys. Rev. A 69, 043615 (2004).

[18] Y. Hasegawa, S. Menhart, R. Meixner, and G. Badurek, Phys. Lett. A 234, 322 (1997) 
[19] Y. Hasegawa, and G. Badurek, Phys. Rev. A 59, 4614 (1999)

[20] A.G. Wagh, G. Badurek, V.C. Rakhecha, R.J. Buchelt, A. Schricker, Phys. Lett. A 268, $209(1997)$

[21] J. Klepp, S. Sponar, Y. Hasegawa, E. Jericha,and G. Badurek, Phys. Lett. A 34248 (2005). 